\title{
Estado nutricional de pacientes com neoplasia de cabeça e pescoço em tratamento oncológico em um hospital público do oeste catarinense
}

Nutritional state of patients with neoplasia of head and neck in oncologicall treatment in a public hospital in west catarinian

Estado nutricional de pacientes con neoplasia de cabeza y cuello en tratamento oncológico en un hospital público de catarina occidental

Recebido: 21/04/2021 | Revisado: 30/04/2021 | Aceito: 02/05/2021 | Publicado: 16/05/2021

Keli Raquel Secchi

ORCID: https://orcid.org/0000-0001-6837-5691 Associação Hospitalar Lenoir Vargas Ferreira, Brasil E-mail: kelisecchi@unochapeco.edu.br

Rosana Amora Ascari

ORCID: https://orcid.org/0000-0002-2281-8642

Universidade do Estado de Santa Catarina, Brasil E-mail: rosana.ascari@hotmail.com

Fernanda Ceconello

ORCID: https://orcid.org/0000-0001-5030-5708 Hospital Regional do Oeste, Brasil E-mail: ccpfernanda@gmail.com

Junir Antonio Lutinski

ORCID: https://orcid.org/0000-0003-0149-5415 Universidade Comunitária da Região de Chapecó, Brasil E-mail: junir@unochapeco.edu.br

Daniele Tiepo

ORCID: https://orcid.org/0000-0001-7585-7218 Associação Hospitalar Lenoir Vargas Ferreira, Brasil E-mail: danitiepo@unochapeco.edu.br

Jaine Buzzetti

ORCID: https://orcid.org/0000-0001-5069-8345 Associação Hospitalar Lenoir Vargas Ferreira, Brasil E-mail: jaine.buzzetti@unochapeco.edu.br

Daniela Carla Alberti

ORCID: https://orcid.org/0000-0003-4842-2926 Hospital da Criança Augusta Muller Bonher, Brasil E-mail: danielaalberti@unochapeco.edu.br

\begin{abstract}
Resumo
Objetivo: Analisar o estado nutricional de pacientes com câncer de cabeça e pescoço em tratamento oncológico clínico e cirúrgico em um hospital público de referência oncológica no oeste catarinense. Metodologia: Estudo transversal, exploratório e descritivo de abordagem quantitativa, desenvolvido em um hospital público no oeste catarinense com 35 pacientes diagnosticados com neoplasia maligna de cabeça e pescoço em tratamento oncológico no local. A coleta dos dados foi realizada entre os meses de março a agosto de 2020. Resultados: A maioria era do sexo masculino (88,6\%), com média de idade de 59,5 anos, possuíam o ensino fundamental I incompleto, casados e com filho. A prevalência de diagnóstico foi o carcinoma espinocelular de orofaringe. Em relação a ASG-PPP a supremacia dos pacientes manifestou que a ingesta alimentar no último mês está menor que o normal e apresentam um ou mais sintomas que impedem de comer o suficiente nas duas últimas semanas. Ainda apresentaram em sua maioria algum grau de desnutrição, e necessitam de avaliação e intervenção nutricional em algum grau de complexidade. A antropometria, contudo, aponta eutrofia $(48,8 \%)$ conforme IMC, e as demais medidas indicam déficit ou risco de déficit de tecidos condizentes ao encontrado na ASG-PPP. Os fatores mais frequentes que interferem na alimentação foram boca seca e alteração de paladar. Conclusão: Conclui-se que os pacientes com neoplasia de cabeça e pescoço necessitam de algum grau de intervenção e/ou orientação nutricional, baseado nos inúmeros sintomas que apresentam, prevalência de quadros de desnutrição, bem como alteração de consumo alimentar.
\end{abstract}

Palavras-chave: Avaliação nutricional; Neoplasia de cabeça e pescoço; Serviço hospitalar de oncologia. 


\begin{abstract}
Objective: To analyze the nutritional status of patient's with head and neck cancer clinical and surgical cancer treatment at a public oncology referral hospital in western Santa Catarina. Methodology: Cross-sectional, exploratory and descriptive study with a quantitative approach, developed in a public hospital in western Santa Catarina with 35 patients diagnosed with malignant neoplasm of the head and neck undergoing oncological treatment at the site. Data collection was carried out between March and August 2020. Results: Most of them were male (88.6\%), with na average age of 59.5 years old, had incomplete elementary school, married and with son. The prevalence of diagnosis was oropharyngeal squamous cell carcinoma. Regarding ASG-PPP, the supremacy of the patients showed that the food intake in the last month is lower than normal and they present one or more symptoms that prevent them from eating enough in the last two weeks. Most of them still presented some degree of malnutrition, and need nutritional assessment and intervention in some degree of complexity. Anthropometry, however, points to eutrophic (48.8\%) according to BMI, and the other measures indicate deficit or risk of deficit of tissues consistent with that found in the ASG-PPP. The most frequent factors that interfere with food were dry mouth and changes in taste. Conclusion: It is concluded that patients with head and neck cancer need some degree of intervention and/or nutritional guidance, based on the numerous symptoms they present, prevalence of malnutrition, as well changes in food consumption.
\end{abstract}

Keywords: Nutritional assessment; Head and neck neoplasm; Hospital service oncology.

\title{
Resumen
}

Objetivo: Analizar el estado nutricional de pacientes con cáncer de cabeza y cuello sometidos a tratamiento oncológico clínico y quirúrgico en un hospital público de referencia de oncología del occidente de Santa Catarina. Metodología: estudio transversal, exploratorio y descriptivo con abordaje cuantitativo, desarrollado en un hospital público del occidente de Santa Catarina con 35 pacientes diagnosticados de neoplasia maligna de cabeza y cuello sometidos a tratamiento oncológico en el sitio. La recolección de datos se realizó entre marzo y agosto de 2020. Resultados: La mayoría eran hombres (88.6\%), con una edad promedio de 59.5 años, tenían primaria incompleta, casados y con hijo. La prevalencia del diagnóstico fue carcinoma de células escamosas de orofaringe. En cuanto a ASG-PPP, la supremacía de los pacientes mostró que la ingesta de alimentos en el último mes es menor de lo normal y presentan uno o más síntomas que les impiden comer lo suficiente en las últimas dos semanas. La mayoría de ellos todavía presenta algún grado de desnutrición, y necesitan evaluación e intervención nutricional en cierto grado de complejidad. La antropometría, sin embargo, apunta a la eutrofia $(48,8 \%)$ según el IMC, y las otras medidas indican déficit o riesgo de déficit de tejidos consistente con lo encontrado en el ASG-PPP. Los factores más frecuentes que interfieren con la alimentación fueron sequedad de boca y alteraciones del gusto. Conclusión: Se concluye que los pacientes con cáncer de cabeza y cuello necesitan algún grado de intervención y / o orientación nutricional, en función de los numerosos síntomas que presentan, prevalencia de desnutrición, así como cambios en el consumo de alimentos.

Palabras clave: Evaluación nutricional; Neoplasia de cabeza y cuello; Servicio de oncología hospitalaria.

\section{Introduçãa}

A denominação câncer se deve a um conjunto de doenças que possuem como característica o crescimento celular desordenado que invade órgãos e tecidos (Instituto Nacional de Câncer José Alencar Gomes da Silva, 2019a). Entre os tipos de câncer que podem se desenvolver estão as neoplasias malignas de cabeça e pescoço, localizadas no trato aerodigestivo superior, as quais possuem como histologia constate o carcinoma espinocelular ou escamoso (CEC) (Chaves, Soares, Kawalski, Santos \& Oliveira, 2020). Dentro desta localização se enquadram os cânceres de boca ou câncer de lábios e cavidade oral, câncer de laringe, câncer de orofaringe, câncer de hipofaringe e câncer de nasofaringe de forma mais frequente (Instituto Nacional de Câncer José Alencar Gomes da Silva, 2019b).

A desnutrição no paciente oncológico adulto ocorre devido a múltiplos fatores, como a ingestão alimentar inadequada agravada pelo aumento das necessidades e danos na absorção e utilização de nutrientes, além da resposta imunoinflamatória que ocasiona o hipercatabolismo e este por sua vez intensifica a perda de massa magra e mudanças na imunocompetência, ambas relacionadas a desfechos negativos (Instituto Nacional de Câncer José Alencar Gomes da Silva, 2016). Além desses fatores, a localização das neoplasias malignas de cabeça e pescoço e o tratamento sobre estas estruturas acometidas intensificam os prejuízos sobre o estado nutricional, podendo resultar em impactos negativos na qualidade de vida, morbidade e mortalidade (Oliveira et al, 2015).

Assim, o acompanhamento nutricional como parte do tratamento do paciente oncológico de forma individualizada, desde a avaliação nutricional, o cálculo das necessidades nutricionais, a terapia nutricional objetiva aumentar a ingestão 
calórico-proteica, peso corporal e melhorar a composição corporal, o que consequentemente resulta em melhor resposta ao tratamento, reduz os riscos infecciosos pós-operatórios e morbimortalidade, além de auxiliar no controle dos sintomas relacionados aos tumores (Santos, Corrêa, Faria, Reis \& Pinheiro, 2019).

Com base no exposto, questiona-se: Qual o estado nutricional de pacientes com câncer de cabeça e pescoço em tratamento oncológico em um hospital público de referência oncológica no oeste catarinense? Que fatores interferem na alimentação de pacientes com câncer de cabeça e pescoço em tratamento oncológico?

O presente estudo teve por objetivo analisar o estado nutricional de pacientes com câncer de cabeça e pescoço em tratamento oncológico clínico e cirúrgico em um hospital público de referência oncológica no oeste catarinense.

\section{Metodologia}

Trata-se de um estudo transversal, exploratório e descritivo de abordagem quantitativa, realizado com 35 pacientes em tratamento oncológico cirúrgico e/ou clínico de um hospital público de referência oncológica no oeste catarinense, no período de março a agosto de 2020. O estudo descritivo busca descrever os fatos e fenômenos de uma realidade, somado-se ao exploratório, que visa esclarecer e modificar conceitos partindo da instigação destes fenômenos (Gerhardt \& Silveira, 2009). Analisar a exposição destes fatos e fenômenos dentro de um intervalo de tempo ou em um dado momento não determinado se define por transversal (Hochmann, Nahas, Oliveira \& Ferreira, 2005). A pesquisa foi aprovada pelo comitê de ética e pesquisa envolvendo seres humanos sob parecer número 3.736.598, CAAE: 25512019.3.0000.0116, seguindo as resoluções 466/2012 e 510/2016 do Conselho Nacional de Saúde, que determinam as diretrizes e normas regulamentadoras de pesquisa envolvendo seres humanos.

Foram incluídos pacientes oncológicos com idade igual ou superior a 18 anos, de ambos os sexos, com diagnóstico de neoplasia de cabeça e pescoço em tratamento oncológico clínico e/ou cirúrgico. A inclusão deu-se mediante autorização do paciente com assinatura do Termo de Consentimento Livre e Esclarecido (TCLE).

Foram excluídos pacientes oncológicos com neoplasias em outros segmentos anatômicos que não de cabeça e pescoço ou que por algum motivo não tenham sido identificados pelos pesquisadores.

Os dados foram coletados de acordo com a organização dos seguintes blocos: Bloco A (formulário para caracterização da amostra contendo Dados Sociodemográficos), Bloco B (Questionário validado intitulado: Avaliação Subjetiva Global Produzida pelo Paciente ASG-PPP, versão em português) (Gonçalves, Borges, Silveira, Assunção, Orlandi, 2010), Bloco C (Índices antropométricos) e Bloco D (Fatores que interferem na aceitação da alimentação).

O formulário referente aos dados sociodemográficos construído pelos pesquisadores, contempla as seguintes variáveis: sexo, idade, escolaridade, situação conjugal, números de filhos, município de origem, diagnóstico, tempo do diagnóstico e recidiva da doença.

A ASG-PPP avalia o estado nutricional com base na alteração de peso, ingestão de alimentos e sintomas gastrointestinais, capacidade funcional, exame físico e a presença de condições catabólicas, classificando o estado nutricional em três categorias, além de originar a identificação de risco nutricional pelo valor do escore numérico encontrado (Gonçalves, Borges, Silveira, Assunção, Orlandi, 2010; Brasil, 2013).

Os índices antropométricos incluíram medidas antropométricas aferidas de peso e estatura, Índice de Massa Corporal (IMC), Circunferência do Braço (CB), Prega Cutânea Tricipital (PCT), e Circunferência Muscular do Braço (CMB), classificados conforme referências validadas.

O questionário referente aos fatores que interferem na aceitação da alimentação, foi composto pela indagação dos sintomas que interferem o consumo, dificuldade de mastigação e deglutição, uso de via auxiliar de alimentação e alteração de consistência dos alimentos. 
Os dados foram tabulados em planilha do Excel® e posteriormente importados para o Programa Statistical Package of Social Sciences (SPSS) para as análises estatísticas. As variáveis foram exploradas quanto a medidas de posição e dispersão (média e desvio padrão) e atreves de frequências. A natureza da distribuição das variáveis foi verificada com o teste de Shapiro-Wilk. Para avaliar a diferença entre subgrupos das variáveis foi utilizado o teste Anova seguido do pós teste (Post hoc) de Turkey. Foi utilizado o teste de Spearman para verificar possíveis correlações entre variáveis. O nível de significância adotado neste estudo foi de $5 \%(\mathrm{p}<0,05)$ e as análises foram realizadas no programa SPSS versão $22.0 ®$.

\section{Resultados}

Participaram deste estudo 35 pacientes com média de idade de 59,5 anos, variando entre 26 e 80 anos. A maioria dos participantes possuíam o ensino fundamental I completo/incompleto $(\mathrm{n}=21 ; 60 \%)$, seguido de ensino fundamental II $(\mathrm{n}=7$; $20 \%)$, ensino médio $(n=4 ; 11,4 \%)$ e ensino superior $(n=3 ; 8,6 \%)$, eram casados $(n=24 ; 68,6 \%)$ e com filhos ( $n=32 ; 91,4 \%)$. Houve predomínio do sexo masculino com 31 participantes $(88,6 \%)$.

Dentre os cânceres de cabeça e pescoço houve prevalência de diagnóstico de carcinoma espinocelular de orofaringe com nove casos (25,7\%), consecutivo de carcinoma espinocelular de laringe com sete (20,0\%), carcinoma espinocelular de língua com cinco $(14,4 \%)$, carcinoma escamoso de hipofaringe com três $(8,6 \%)$, carcinoma espinocelular de cavidade oral com dois $(5,7 \%)$, carcinoma de mandíbula com dois $(5,7 \%)$, carcinoma indiferenciado de nasofaringe com dois (5,7\%), o carcinoma de assoalho de boca, carcinoma escamoso de gengiva, carcinoma de prega ariepiglótica, neoplasia maligna submandibular e a neoplasia maligna de glândula parótida com um caso cada (2,9\%). Já o tempo de diagnóstico foi de menos de um ano (71,4\%), caracterizado por recidiva $(25,7 \%)$.

A aplicação do instrumento da avaliação subjetiva global produzida pelo próprio paciente (ASG-PPP) resultou na média de massa de $63,1 \mathrm{~kg}$ e altura de $168,9 \mathrm{~cm}$. O peso médio há um mês e seis meses correspondeu a $65,5 \mathrm{~kg}$ e $69,8 \mathrm{~kg}$, respectivamente, indicando perda de massa antecedente ao diagnóstico. Entretanto, a maioria dos entrevistados ( $\mathrm{n}=17 ; 48,6 \%)$ julgou ter mantido o peso durante as duas últimas semanas, a diminuição foi encontrada em $n=12(34,3 \%)$ participantes, sendo o aumento menos frequente $(\mathrm{n}=6 ; 17,1 \%)$. A perda de peso média em seis meses em comparação ao atual foi de $6,7 \mathrm{~kg}$.

A ingesta alimentar no último mês foi menor que o normal e teve supremacia em $n=23(65,7 \%)$ participantes, ficando a consistência líquida e sólida em menor quantidade $(\mathrm{n}=8 ; 22,9 \%)$ cada, enquanto sólidos em pouca quantidade e apenas alimentos por sonda ou veia ambos corresponderam a cinco respostas $(14,3 \%)$.

Quando questionados sobre a presença de sintomas/problemas durante as duas últimas semanas que impedem de comer o suficiente, a apresentação de um ou mais sintomas foi frequente. Dentre os sintomas assinalados prevaleceu a dor em $\mathrm{n}=22$ pacientes $(62,9 \%)$, sensação de que os alimentos têm gosto estranho $(\mathrm{n}=20 ; 57,1 \%)$, boca seca $(\mathrm{n}=20 ; 57,1 \%)$, enjoos a cheiros $(n=18 ; 51,4 \%)$, problemas para engolir $(n=17 ; 48,6 \%)$, falta de apetite $(n=16 ; 45,7)$, feridas na boca $(n=14 ; 40,0 \%)$, constipação $(n=12 ; 34,6 \%)$, náuseas $(n=11 ; 31,4 \%)$, diarreia $(n=7 ; 20,0 \%)$, vômito $(n=6 ; 17,1 \%)$ e rapidamente me sinto satisfeito $(\mathrm{n}=2 ; 5,7 \%)$. Apenas dois participantes $(5,7 \%)$ afirmaram que não possuíam problemas para se alimentar.

Em relação a dor, as localizações citadas foram garganta ( $n=6 ; 17,1 \%)$, abdômen $(n=4 ; 11,4 \%)$, pescoço e mandíbula $(\mathrm{n}=2 ; 5,7 \%)$, boca, ouvido, perna, ombros, língua e cabeça $(\mathrm{n}=1 ; 2,9 \%)$ cada. A citação de dor em mais de uma localização foi frequente $(27,2 \%)$. Outros sintomas que impediam de comer o suficiente nas duas últimas semanas foram sensibilidade nos dentes, a temperatura e doces, tosse, ardência na língua, excesso e falta de saliva, alteração de voz e problemas dentários, este último sinalizado por dois participantes $(5,7 \%)$.

A triagem nutricional realizada por meio do instrumento ASG-PPP apontou predomínio do escore mais alto, sugerindo intervenção (Tabela 1). 
Tabela 1 - Panorama da triagem nutricional de pacientes com neoplasia de cabeça e pescoço em tratamento oncológico segundo ASG-PPP. Chapecó - SC, Brasil, 2020. (n=35).

\begin{tabular}{lc}
\hline \multicolumn{1}{c}{ Variável } & $\mathrm{N}(\%)$ \\
\hline $\mathbf{2}$-3 (pontos) Educação do paciente e seus familiares pelo nutricionista, enfermeira ou & $1(2,9)$ \\
outro profissional, com intervenção farmacológica de acordo com o inquérito de & \\
sintomas (caixa 3) e exames laboratoriais se adequado & $7(20,0)$ \\
$\mathbf{4 - 8}$ (pontos) Necessita de intervenção pelo nutricionista, juntamente com enfermeira & \\
ou médico conforme o inquérito de sintomas (caixa 3) & $27(77,1)$ \\
>9 (pontos) Indica necessidade crítica de melhora no manuseio dos sintomas e/ou \\
opções de intervenção nutricional
\end{tabular}

Fonte: Banco de dados dos Autores (2020).

Em relação ao estado nutricional, os pacientes com neoplasia de cabeça e pescoço apresentaram em sua maioria algum grau de desnutrição, seja, desnutrição moderada ou suspeita de desnutrição $(n=19 ; 54,3 \%)$ ou gravemente desnutrido (n=8; 22,9\%). Menos de um quarto dos participantes encontram-se bem nutridos ou anabólicos (n=8; 22,9\%).

Os índices antropométricos indicaram média de peso aferido de 43,1 kg, com um desvio padrão de $28,2 \mathrm{~kg}$, a estatura por sua vez teve média de $168 \mathrm{~cm}$, e IMC 22,2 kg/m². Deste modo, pela classificação do IMC considerando a classificação para idade, o baixo peso que corresponde a desnutrição em idosos (acima de 60 anos) e a desnutrição independente do grau em adultos não apontou prevalência como indicado na ASG-PPP, sendo prevalente a eutrofia. Contudo, as classificações da circunferência do braço $(\mathrm{CB})$ e da prega cutânea tricipital (PCT) apresentaram domínio de déficit de gordura ou risco de déficit de gordura em comparação a normalidade.

A circunferência muscular do braço (CMB) que associa em equação a CB e a PCT para avaliar a musculatura, apontou em sua maioria algum grau de perda de reservas musculares, seja déficit ou baixa de massa magra.

Referente aos fatores que interferem na alimentação, a boca seca e alteração de paladar foram os mais frequentes $(\mathrm{n}=18 ; 51,4 \%$ cada), seguido de constipação $(\mathrm{n}=11 ; 31,4 \%)$, falta de apetite $(\mathrm{n}=11 ; 31,4 \%)$ e náuseas/enjoo $(\mathrm{n}=10 ; 28,6 \%)$, enquanto os menos frequentes foram vômito $(n=4 ; 11,4 \%)$ e diarreia $(n=3 ; 8,6 \%)$.

A dor em diversas regiões do corpo, como a abdominal, ao deglutir a saliva, mastigar, garganta, boca, costas e cabeça foram frequentemente observada nos pacientes do estudo. Também sintomas de tontura, fraqueza e sono que indicam indisposição também foram verificados. Além do mais, alguns dos sintomas citados ou até mesmo todos são apontados como interferência na vontade de se alimentar.

Apesar de a menor parcela fazer uso de sonda de forma exclusiva ou associada a via oral $(25,3 \%)$, a maioria (82,9\%) adaptou a consistência dos alimentos para o consumo após o diagnóstico, $60 \%$ declararam dor para mastigar ou engolir os alimentos e 76,1\% relataram dificuldades de realizar esse processo.

Assim, a associação de sintomas decorrentes do tratamento oncológico (Escore sintomas ASG-PPP) com o estado

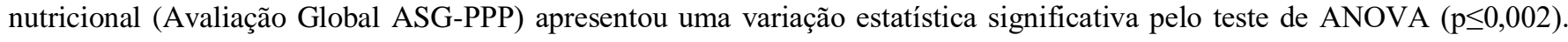
Pacientes que apresentavam mais sintomas tiveram algum grau de comprometimento do estado nutricional.

Foi encontrada diferença significativa dos resultados entre bem nutrido ou anabólico com desnutrição moderada e grave ( $\mathrm{p} \leq 0,004$ e $\mathrm{p} \leq 0,003)$. Já a desnutrição grave não diferenciou dos resultados de desnutrição moderada $(\mathrm{p} \leq 0,732)$.

$\mathrm{Na}$ interpretação da análise estatística de Spearman o coeficiente resultante entre a variável escore dos sintomas apresentou correlação com a variável avaliação global ( $r s=0,492 ; \mathrm{p}=1,000)$, comprovando que são perfeitamente lineares e há uma forte relação entre as variáveis. 


\section{Discussão}

A partir dos dados coletados da caracterização sociodemográfica, observa-se que o serviço tem prevalência de público masculino com diagnóstico de neoplasia maligna de cabeça e pescoço. O resultado corrobora com dados do Instituto Nacional do Câncer José Alencar Gomes da Silva (2019c) em que os tumores de cabeça e pescoço representaram a segunda maior incidência de neoplasias em homens brasileiros em 2019.

Os tipos de câncer mais frequentes em homens previstos para 2020 no Brasil, à exceção do câncer de pele não melanoma, o de cavidade oral corresponde à $5^{\text {a }}$ posição e o câncer de laringe em $7^{\circ}$ lugar. Em se tratando do câncer de cavidade, Santa Catarina por sua vez, está entre os estados brasileiros com mais casos deste diagnóstico (Instituto Nacional de Câncer José Alencar Gomes da Silva, 2019d). No presente estudo, o câncer de cavidade oral somado a outros diagnósticos específicos que o compõe (língua, gengiva, assoalho de boca) corresponde a 25,9\% dos casos.

O tempo de diagnóstico de câncer entre os participantes com recidiva da doença nesse estudo foi superior a 14 meses, sendo que quanto menor foi o tempo de diagnóstico menor a ocorrência de recidiva. O prognóstico depende do estágio do tumor no diagnóstico e do local de envolvimento, sendo a sobrevida global de cinco anos em pacientes com doença em estágio I ou II e menor nos estágios III ou IV. Além disso, possuir fatores de risco contribui com tal desfecho, como a associação ao tabaco, álcool e a presença ou não do papilomavírus humano (HPV) em alguns tipos de neoplasias malignas de cabeça e pescoço (Haddad \& Limaye, 2020).

Ao comparar os resultados de pesos referidos na ASG-PPP com os aferidos pelo instrumento dos índices antropométricos, observou-se diferença de média dos pesos, onde o referido foi de $63,1 \mathrm{~kg}$ e o aferido e $43,1 \mathrm{~kg}$, ressaltando a importância da aferição antes de prescrição de medicações, principalmente quimioterápicos antineoplásicos, uma vez que equívocos resultam em efeitos colaterais graves. Atenta-se para variáveis como altura, peso e superfície corporal, importantes na segurança do paciente na administração de quimioterapia antineoplásica, pois estão diretamente relacionados à dosagem do medicamento e erros em uma ou mais etapas do processo podem induzir toxicidades e até mesmo, o óbito (Ribeiro \& Santos, 2015).

A perda involuntária de peso também está associada ao aumento da toxicidade do tratamento, complicações e permanência hospitalar (Cagol et al, 2016). Entre suas causas encontra-se a ingestão alimentar insuficiente, a localização da neoplasia maligna, o avanço da patologia e o próprio tratamento quimioterápico (Milani, Pereira, Barbosa \& Barichello, 2018).

A sensação de boca seca ou xerostomia é referida constantemente pelos pacientes de câncer de cabeça e pescoço, principalmente os que se submeteram à radiação como parte do tratamento (Haddad \& Limaye, 2020), sintomas que podem ser prevenidos por meio da menor exposição das glândulas salivares à radiação, adaptações na dieta como inclusão de alimentos úmidos e mais macios, substâncias ácidas ou amargas e aumento na frequência de consumo de água (Galloway \& Amdur, 2020). A dor oncológica pode ser resultante de efeitos do tumor ou por complicações do tratamento e causar impacto na qualidade de vida, interferindo na funcionalidade física, psicológica e social devido a mudanças no sono e humor (Vieira, Brás \& Fragoso, 2019).

A prevalência de desnutrição nos pacientes oncológicos variou conforme o método de avaliação (ASG e medidas antropométricas). Em um estudo com pacientes com câncer de cavidade oral em pré-tratamento antineoplásico, a prevalência de desnutrição também variou dependendo do método utilizado para avaliação do estado nutricional (Oliveira et al, 2015). Com a utilização do instrumento ASG-PPP como método de coleta, pesquisadores informam suspeita de desnutrição e desnutrição moderada ou grave em 59,5\% em pacientes com câncer de cabeça e pescoço em quimioterapia (Gomes \& Maio, 2015).

A desnutrição é frequente nos indivíduos com câncer e tem como possíveis causas as alterações no metabolismo provocadas pela doença e tratamento, além da redução na ingestão total e o aumento da demanda energética pela evolução do 
tumor, que culmina num estado nutricional deficiente e, consequentemente, influencia na evolução do paciente oncológico (Milani, Pereira, Barbosa \& Barichello, 2018). A alteração de consistência dos alimentos apresentada pela maioria dos participantes, se assemelha com outro estudo em que 89,9\% dos entrevistados afirmaram alterações na consistência das preparações de consumo (Gomes \& Maio, 2015). Em uma revisão sistemática, todos os estudos analisados apresentaram alterações na deglutição dos pacientes antes e após o tratamento, emergindo a necessidade de uso de via auxiliar de alimentação, redução da ingestão oral e restrições dietéticas e disfagia (Gois, Barbosa, Matos, Cesar \& Paranhos, 2020).

A indicação de intervenções pelos escore da ASG-PPP no atual estudo, indicou na maioria dos casos, a necessidade crítica de intervenção nutricional, assemelhando-se aos dados da literatura em que mais da metade da amostra possuía necessidade crítica intervenção nutricional (Guilherme, Bastos, Casado \& Burgos, 2020). A condição nutricional é dependente do tipo e localização do tumor, de modo que indivíduos com neoplasias que interferem na ingestão alimentar estão mais sujeitos a comprometimentos do estado nutricional (Cagol et al, 2016). Assim, o suporte nutricional precoce parece melhorar o desfecho por colaborar na manutenção do peso corporal, evitar a deterioração do estado nutricional e prevenir a desnutrição (Exposíto et al, 2018; Brazilian society of parenteral and enteral nutrition, 2019).

Assim, a indicação de suplementos orais ou alimentação por sonda para pacientes com câncer de cabeça e pescoço deve ser iniciada precocemente considerando o estado nutricional, dados demográficos, local e estadiamento do tumor, disfagia, morbidades, impacto das modalidades de tratamento e a capacidade do paciente de manter ou atingir as necessidades nutricionais (Brazilian society of parenteral and enteral nutrition, 2019).

A apresentação de sintomas que interferem na ingesta alimentar com o estado nutricional deficiente, comprova a necessidade da oferta adequada de nutrientes. Assim, os achados despertam para a necessidade de olhar atento dos profissionais na tomada de decisão e definição de condutas, uma vez que a maioria dos participantes não faziam uso de via auxiliar, mesmo confirmando a indicação pela alteração de consumo alimentar. Estudo constatou algum grau desnutrição pela ASG-PPP $(62,2 \%)$ em pacientes com câncer de cabeça e pescoço, sendo que destes, quase todos (98\%) apresentavam algum sintoma que impedia de se alimentar adequadamente (Aldama, Ortíz, Joy \& Morínigo, 2016).

Prevenir a desnutrição evita prejuízos nas funções imunológicas, reduz a morbidade e melhora a resposta terapêutica ao tratamento (Weissheimer, Rech, 2017). Portanto, incluir a terapia nutricional em pacientes oncológicos se torna indispensável para preservar o estado nutricional e permitir o bom funcionamento dos sistemas orgânicos vitais.

Faz-se necessário novos estudos acerca do tipo de tratamento e presença de sintomas com estado nutricional e seus desfechos.

\section{Conclusão}

Há necessidade de intervenção e/ou orientação nutricional com base nos achados de prevalência de quadros de desnutrição, sintomas apresentados, alteração de consumo alimentar, perda de peso, bem como nos problemas para deglutir os alimentos. A avaliação nutricional precoce de pacientes com câncer de cabeça e pescoço, clínico ou cirúrgico, bem como a correta intervenção nutricional, pode contribuir para minimizar os danos decorrentes estado nutricional deficiente antes mesmo do início do tratamento oncológico.

Este estudo teve algumas limitações como o tamanho pequeno da amostra, a ausência de dados relacionados à fase do tratamento. Recomenda-se para trabalhos futuros a incorporação de maior amostra, incluir dados detalhados das fases de tratamento, bem como estratificar a modalidade de tratamento principalmente associado aos sintomas apresentados.

\section{Referências}

Aldama, L. G., Ortíz, L. P., Joy, L., \& Morínigo, M. (2016). Desnutrición a partir de la valoración global subjetiva generada por el paciente (VGS-GP) en pacientes con cáncer de cabeza y cuello. Memorias del Instituto de Investigaciones em Ciencias de la Salud. 14(1), 86-93. 
Research, Society and Development, v. 10, n. 5, e53710515294, 2021

(CC BY 4.0) | ISSN 2525-3409 | DOI: http://dx.doi.org/10.33448/rsd-v10i5.15294

Brasil (2013). Ministério da Saúde. Instituto Nacional de Câncer. Inquérito brasileiro de nutrição oncológica. INCA.

Brazilian society of parenteral and enteral nutrition (2019). Diretriz BRASPEN de terapia nutricional no paciente com câncer. Braspen Journal. $34(1)$, 2-32.

Cagol, F., Pretto, A. D. B., Colling, C., Araújo, E. S., Vale, I. A. V., Bergmann, R. B., Pastore, C. A., \& Moreira, A. N. (2016). Estado nutricional segundo avaliação subjetiva global produzida pelo paciente de acordo com a localização do tumor. Nutricíon Clínica Dietética e Hospitalaria.36(4), 13-9.

Chaves, A. L. F., Soares, J. M. A., Kawalski, L. P., Santos, M., \& Oliveira, T. B. (2020). Cabeça e pescoço: Doença localizada e localmente avançada. SBOC.

Exposíto, M. R. A., Herrera-Martínez, A. D., García, G. M., Calvo, M. E., Serrano, C. M. B., \& Moreno, M. A. G. (2018). Early nutrition support therapy in patients with head-neck cancer. Nutrição hospitalar. 35(1), 505-10.

Galloway, T., Amdur, R. J. (2020). Management of late complications of head and neck cancer and its treatment. http://www.uptodate.com/online

Gerhardt, T. E., Silveira, D. T. (2009). Métodos de pesquisa. UFRGS editora.

Gois, J. N., Barbosa, A. S., Matos, F. R., Cesar, C. H., \& Paranhos, L. R. (2020). Manifestações da deglutição em pacientes com câncer de orofaringe submetidos à terapia conservadora: revisão sistemática. Jornal Brasileiro de Patologia e Medicina Laboratorial. 56(1), 1-11.

Gomes, N. S., \& Maio, R. (2015). Avaliação subjetiva global produzida pelo próprio paciente e indicadores de risco nutricional no paciente oncológico em quimioterapia. Revista Brasileira de Cancerologia. 61(3), 235-42.

Gonçalves, M. C., Borges, L. R., Silveira, D. H., Assunção, M. C. F., \& Orlandi, S. P. (2010). Validação da versão em português da avaliação subjetiva global produzida pelo paciente. Revista Brasileira de Nutrição Clínica. 25(2), 102-8.

Guilherme, L. G., Bastos, L. L. S., Casado, A. H. S., \& Burgos, M. G. P. A. (2020). Terapia nutricional em pacientes oncológicos: realidade de um hospital de referência em Pernambuco. Nutricíon clínica dietética e Hospitalaria. 40(1), 33-9.

Haddad, R., \& Limaye, S. (2020). Overview of approach to long-term survivors of head and neck câncer. http://www.uptodate.com/online

Hochmann, B., Nahas, F. X., Oliveira, R. S. Filho, Ferreira, L. M. (2005). Desenhos de pesquisa. Acta cirurgica brasileira. $20(2), 1-9$.

Instituto Nacional de Câncer José Alencar Gomes da Silva, (2016). Consenso Nacional de Nutrição Oncológica. (2a ed.), INCA.

Instituto Nacional de Câncer José Alencar Gomes da Silva. (2019a). O que é câncer? https://www.inca.gov.br/o-que-e-cancer

Instituto Nacional de Câncer José Alencar Gomes da Silva, (2019b). Tipos de Câncer. https://www.inca.gov.br/tipos-de-cancer

Instituto Nacional de Câncer José Alencar Gomes da Silva (2019c). Julho Verde: INCA debate linha de cuidado e diagnóstico precoce de tumores de cabeça e pescoço. https://www.inca.gov.br/noticias/julho-verde-inca-debate-linha-de-cuidado-e-diagnostico-precoce-de-tumores-de-cabeca-e

Instituto Nacional de Câncer José Alencar Gomes da Silva (2019d). Estimativa 2020: incidência de câncer no Brasil. INCA.

Milani, J., Pereira, E. M. S., Barbosa, M. H., \& Barichello E. (2018). Antropometria versus avaliação subjetiva nutricional no paciente oncológico. Acta Paulista Enfermagem. 31(3), 240-46.

Oliveira, F. P., Santos, A., Viana, M, S., Alves, J. L., Pinho, N. B., \& Reis, P. F. (2015). Perfil nutricional de pacientes com câncer de cavidade oral em prétratamento antineoplásico. Revista Brasileira de Cancerologia. 61(3), 253-59.

Ribeiro, T. S., Santos, V. O (2015). Segurança do Paciente na Administração de Quimioterapia Antineoplásica: uma Revisão Integrativa. Revista Brasileira de Cancerologia. 61(2), 153-58.

Santos, M., Corrêa, T. S., Faria, L. D. B. B., Reis, P. E. D., \& Pinheiro, R. N (2019). Diretrizes oncológicas. (2a ed.), Doctor Press.

Vieira, C., Brás, M., \& Fragoso, M. (2019). Opióides na dor oncológica e o seu uso em circunstâncias particulares: uma revisão narrativa. Acta Medica Portuguesa. 32(5), 388-99.

Weissheimer, A., \& Rech, C. R. A. (2017). O papel da terapia nutricional nos tumores de cabeça e pescoço. Revista Nutrição Vigilância e Saúde. 4(1), 80-6. 